09,12

\title{
Магнитоплазмонные кристаллы: резонансные линейные и нелинейные магнитооптические эффекты
}

\author{
(C) А.Л. Чехов ${ }^{1}$, П.Н. Найденов ${ }^{2}$, О.В. Голикова ${ }^{2}$, А.В. Беспалов ${ }^{2}$, А.И. Стогнийํ, Т.В. Мурзина ${ }^{1}$ \\ ${ }^{1}$ Московский государственный университет им. М.В. Ломоносова, \\ Москва, Россия \\ ${ }^{2}$ Московский технологический университет (МИРЭА), \\ Москва, Россия \\ ${ }^{3}$ НПЦ НАН Беларуси по материаловедению, \\ Минск, Беларусь \\ E-mail: chekhov@shg.ru
}

\begin{abstract}
Представлены результаты исследования спектроскопии генерации второй оптической гармоники (ВГ) в магнитных плазмонных структурах на основе феррита граната и периодических массивов нанополос золота. Экспериментально показано, что в области резонансного возбуждения поверхностного плазмона на границе раздела металла и магнитного диэлектрика наблюдается увеличение интенсивности ВГ, а также знакопеременная модуляция магнитного контраста ВГ, достигающая 40\%. Полученные результаты описаны в терминах нелинейного резонанса Фано.
\end{abstract}

Работа выполнена при поддержке РФФИ (гранты № 16-02-0100, 16-32-00702) и стипендии Президента РФ СП-382.2015.5.

\section{1. Введение}

Магнитоплазмонные кристаллы (МПК), т.е. пространственно-периодические плазмонные гетероструктуры, в состав которых входят магнетики, в последние годы являются объектом активных исследований $[1,2]$. Одним из наиболее перспективных вариантов МПК является структура, состоящая из слоя периодически перфорированного благородного металла (золота) на поверхности пленки магнитного диэлектрика. Наличие в МПК периодической металлической структуры приводит к изменению закона его дисперсии по сравнению со сплошной пленкой металла, в результате чего оказывается возможным эффективное резонансное возбуждение поверхностных плазмон-поляритонов (ППП) за счет выполнения соответствующего условия фазового квазисинхронизма [3,4]. Наличие в структуре МПК магнитного диэлектрика приводит в свою очередь к зависимости условия квазисинхронизма от намагниченности структуры. Таким образом, реализуется возможность управления оптическим и магнитооптическим спектром взаимодействующего с МПК излучения, что преимущественно и определяет интерес к изучению таких структур.

Ранее было показано, что возбуждение в плазмонном кристалле ППП двух видов, соответствующих границам раздела золотой структуры с воздухом и магнитным диэлектриком, а также волноводных мод в магнитной диэлектрической пленке приводит к появлению резонансов в частотно-угловых спектрах пропускания, в окрестности которых наблюдаются ряд особенностей и возрастание линейных магнитооптических эффектов [5-8]. В то же время нелинейно-оптические эффекты в МПК изучены в гораздо меньшей степени, хотя можно ожидать, что помимо эффектов, связанных с дисперсионными свойствами МПК, в нелинейно-оптическом отклике будут проявляться и эффекты локализации оптического поля в пространственной области возбуждения ППП, т.е. на границах раздела с металлической структурой. Наиболее эффективно это может проявиться в нелинейнооптических эффектах четного порядка, в том числе в генерации второй оптической гармоники (ВГ), отличающейся высокой чувствительностью именно к свойствам границ раздела центросимметричных сред [9].

В настоящей работе представлены результаты исследования спектроскопии линейного магнитооптического интенсивностного эффекта и генерации ВГ в магнитных плазмонных структурах на основе периодических массивов нанополос золота, расположенных на пленке висмутзамещенного граната.

\section{2. Методика эксперимента и образцы}

Оптические и нелинейно-оптические эффекты были исследованы в планарных магнитоплазмонных кристаллах, образованных перфорированной пленкой золота (толщиной $40 \mathrm{~nm}$, период МПК $850 \mathrm{~nm}$, ширина полос золота около $730 \mathrm{~nm}$ ) на поверхности слоя висмутзамещенного феррита-граната (BIG) толщиной $2.2 \mu \mathrm{m} \mathrm{c}$ составом $(\mathrm{Bi}, \mathrm{Tm})_{3}(\mathrm{Fe}, \mathrm{Ga})_{5} \mathrm{O}_{12}$, выращенного на подложке галлий-гадолиниевого граната (GGG). Схематическое изображение МПК и геометрии эксперимента приведено на рис. 1,a. Образцы МПК были изготовлены по методике, подробно описанной в [10], основанной на комбинированном ионно-лучевом травлении слоя золота для формирования одномерной пространственнопериодической структуры.

Частотно-угловые спектры пропускания МПК были измерены при использовании стабилизированной галогеновой лампы в качестве источника широкополосного 




Рис. 1. a) Схематическое изображение структуры исследованного магнитоплазмонного кристалла. $b$ ) Спектры пропускания МПК в зависимости от длины волны и угла падения. Показаны области возбуждения ППП на границах раздела воздух/золото (сплошные прямые) и золото/гранат (штриховые прямые).

зондирующего излучения. Пространственная фильтрация излучения осуществлялась с помощью пространственного фильтра, затем зондирующее излучение проходило через поляризатор и фокусировалось на поверхность МПК в пятно диаметром около $50 \mu \mathrm{m}$. Спектры пропускания были нормированы на аналогичные спектры пленки BIG без нанесенной сверху структуры золота. Полоски МПК были ориентированы перпендикулярно плоскости падения пробного излучения. Магнитное поле напряженностью $3 \mathrm{kOe}$, что соответствует полю насыщения пленок граната, было приложено к образцу в поперечной геометрии (рис. 1,a). Образец МПК был помещен на гониометр, что позволяло варьировать угол падения пробного излучения с точностью до долей градуса.

Эксперименты по генерации второй оптической гармоники были выполнены на установке, источником излучения накачки в которой являлся лазер на основе титаната сапфира (диапазон перестройки длин волн 740-860 nm, длительность импульса $100 \mathrm{fs}$, частота следования импульсов $80 \mathrm{MHz}$, средняя энергия излучения $100 \mathrm{~mW}$ ), работающий на длине волны $850 \mathrm{~nm}$. Падающее под углом $10-30^{\circ}$-поляризованное зондирующее излучение фокусировалось на образце в пятно с диаметром около $50 \mu \mathrm{m}$. Излучение ВГ, прошедшее через структуру, выделялось необходимым набором фильтров и регистрировалось фотоэлектронным умножителем Hamamatsu R4220, работающим в режиме счета фотонов.

\section{3. Экспериментальные результаты}

На рис. $1, b$ представлен частотно-угловой спектр пропускания МПК, демонстрирующий набор особенно- стей, преимущественно минимумов пропускания, спектральное положение которых варьируется как функция угла падения $\theta$ и длины волны пробного излучения. Они соответствуют резонансному возбуждению двух видов ППП (на границах раздела воздух/Аu и $\mathrm{Au} / \mathrm{BIG})$, а также волноводных мод в слое граната. Соответствующие спектральные зависимости можно в общем виде описать выражением $k_{i}=k_{0} \sin \theta+m 2 \pi / d$, где индекс $i$ соответствует виду моды, $m \in Z-$ eе порядок, $d$ - период МПК, $k$ и $k_{0}-$ волновые векторы возбужденной моды и падающего излучения соответственно. Горизонтальной линией на рисунке выделена длина волны накачки $850 \mathrm{~nm}$, для которой в дальнейшем проводилось исследование угловых спектров ВГ. Из рис. $1, b$ видно, что вблизи каждой из особенностей зависимости пропускания явно асимметричны и имеют вид резонансов Фано. Подобные особенности имеют место при интерференции резонансного (поле моды, $E_{\mathrm{res}}$ ) и нерезонансного (поле, прошедшее через структуру, не возбудив моду, $E_{\mathrm{nres}}$ полей $I_{\omega} \propto\left[E_{\text {res }}(\theta, \omega)+E_{\text {nres }}(\omega)\right]^{2}$. Спектральные особенности, соответствующие ППП на границе раздела воздух/Аu $(m= \pm 1)$ и $\mathrm{Au} / \mathrm{BIG}(m=2, \pm 3)$ обозначены сплошными и штриховыми линиями соответственно. Особенности, связанные с возбуждением ППП на границе раздела золото/BIG, менее заметны в линейных спектрах, что свидетельствует об их меньшей добротности, которая может быть связана со значительной перекачкой энергии в возбуждение волноводных мод.

На рис. 2 приведены угловые спектры линейного и нелинейного пропускания (на частоте ВГ) МПК для длины волны накачки $850 \mathrm{~nm}$, что соответствует области возбуждения ППП Au/BIG. Видно, что форма спектральной линии в значительной степени асимметрична, 




Рис. 2. Угловые спектры линейного пропускания (штриховая линия) и интенсивности ВГ (точки) в окрестности возбуждения поверхностного плазмона на границе раздела Au/BIG. Длина волны накачки $850 \mathrm{~nm}$.

что указывает на роль интерференционных эффектов в ее формировании. В этом же спектральном диапазоне наблюдается возрастание интенсивности прошедшего через МПК излучения на частоте ВГ со сложной формой спектральной особенности, представляющей собой максимум и два минимума. Асимметричная структура данного резонанса обусловлена наличием нелинейного резонанса Фано [11], при котором отклик нелинейно зависит от поля накачки, обладающего резонансной и нерезонансной составляющими:

$$
I_{2 \omega} \propto\left\{\left[E_{\mathrm{res}}(\theta, \omega)+E_{\mathrm{nres}}(\omega)\right]\left[E_{\mathrm{res}}(\theta, \omega)+E_{\mathrm{nres}}(\omega)\right]\right\}^{2} .
$$

На рис. 3, а представлены частотно-угловые спектры интенсивностного магнитооптического эффекта, наблюдавшегося в линейном оптическом отклике. На рисунке приведены зависимости линейного магнитного контраста, который определен как

$$
\rho_{\omega}=\left\{I_{\omega}(\uparrow)-I_{\omega}(\downarrow)\right\} /\left\{I_{\omega}(\uparrow)+I_{\omega}(\downarrow)\right\},
$$

где $I_{\omega}(\uparrow)$ и $I_{\omega}(\downarrow)$ - интенсивность прошедшего пробного излучения, измеренная для противоположных направлений поперечного магнитного поля. Из приведенных данных видно, что возбуждение ППП на границе раздела $\mathrm{Au} / \mathrm{BIG}$ сопровождается усилением линейного магнитооптического эффекта, максимальная величина которого достигает $0.2 \%$. Следует отметить, что в случае неперфорированной (однородной) магнитной пленки BIG в данной конфигурации эксперимента (в поперечном магнитном поле) не наблюдается магнитного контраста в соответствии с классическим рассмотрением [12]. Нечетный по намагниченности эффект, наблюдавшийся в случае МПК, является следствием чувствительности закона дисперсии ППП на границе раздела $\mathrm{Au} / \mathrm{BIG}$ к намагниченности структуры,

$$
k_{\mathrm{SPP}}=k_{0} \sqrt{\varepsilon_{\mathrm{Au}} /\left(\varepsilon_{\mathrm{Au}}+\varepsilon_{\mathrm{BIG}}\right)}(1+\alpha g)
$$

где $\varepsilon_{\mathrm{Au}}$ и $\varepsilon_{\mathrm{BIG}}$ - диэлектрическая проницаемость золота и граната, $g$ - вектор гирации, $\alpha-$ коэффициент, составленный из диэлектрических проницаемостей [5].
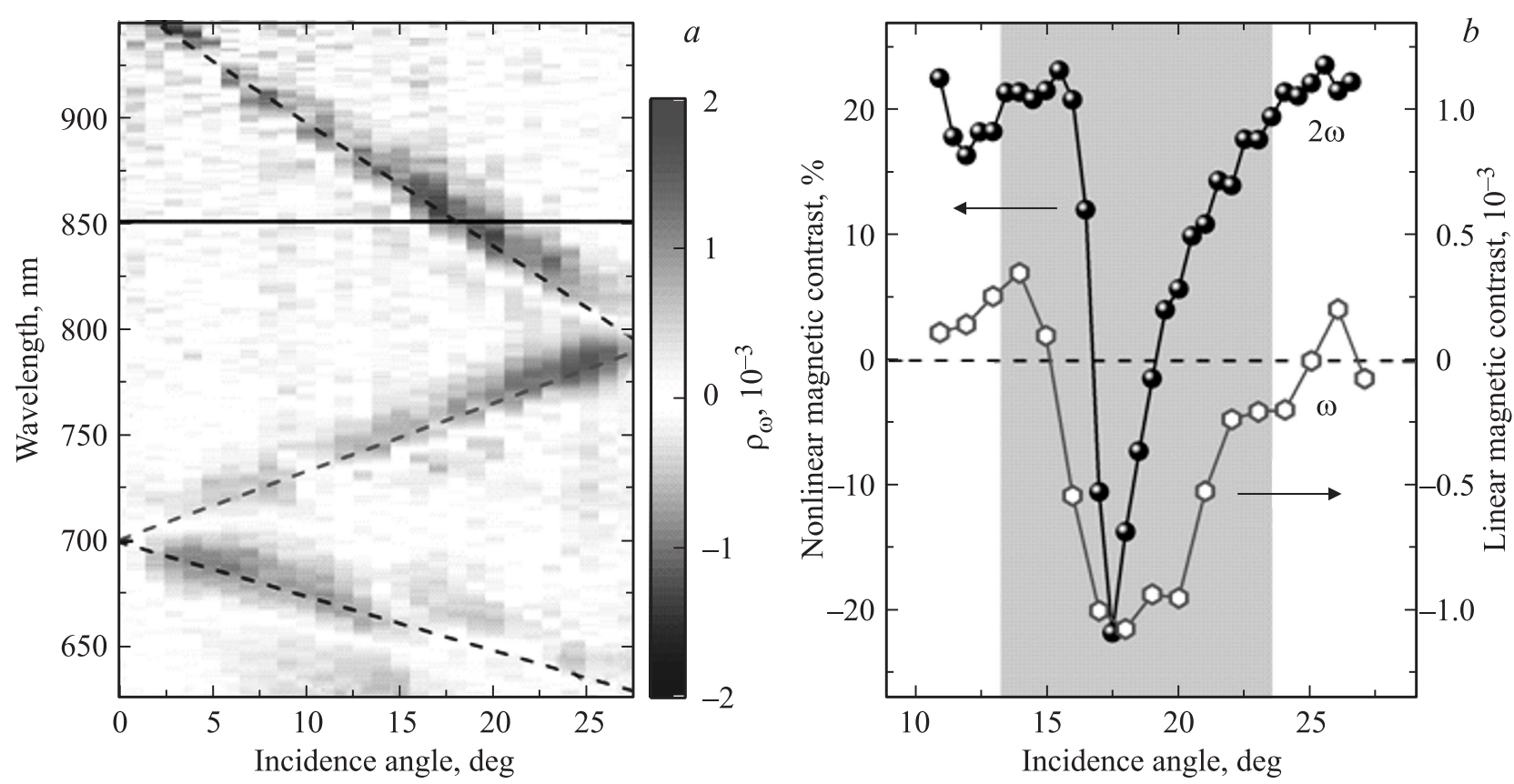

Рис. 3. $a)$ Частотно-угловой спектр линейного магнитного контраста МПК. $b$ ) Угловые спектры линейного (светлые символы) и нелинейного магнитного контраста (темные символы) для длины волны накачки $850 \mathrm{~nm}$. 
В спектральной окрестности возбуждения ППП второго порядка на границе раздела $\mathrm{Au} / \mathrm{BIG}$ были измерены аналогичные спектры магнитного нелинейнооптического интенсивностного эффекта, который также описывается магнитным контрастом

$$
\rho_{2 \omega}=\left\{I_{2 \omega}(\uparrow)-I_{2 \omega}(\downarrow)\right\} /\left\{I_{2 \omega}(\uparrow)+I_{2 \omega}(\downarrow)\right\},
$$

где $I_{2 \omega}(\uparrow)$ и $I_{2 \omega}(\downarrow)$ - интенсивности ВГ для противоположных направлений экваториального магнитного поля. Угловой спектр $\rho_{2 \omega}$, измеренный для длины волны накачки $850 \mathrm{~nm}$, представлен на рис. $3, b$. Видно, что наблюдается знакопеременная зависимость магнитного контраста интенсивности ВГ. Для сравнения на том же рисунке представлен спектр линейного контраста, положение минимума которого совпадает с минимумом зависимости $\rho_{2 \omega}(\theta)$. Видно, что нелинейный контраст обладает большей величиной (на два порядка), а также имеет более резкую резонансную зависимость. С учетом того что поле на частоте ВГ от магнитной нелинейной структуры в общем виде описывается суммой кристаллографической (немагнитной, $\left.\mathbf{E}^{\mathrm{cr}}(2 \omega)\right)$ и магнитоиндуцированной $\left(\mathbf{E}^{\mathrm{M}}(2 \omega)\right)$ составляющих, интенсивность ВГ можно представить в виде $I_{2 \omega} \propto\left\{\mathbf{E}^{\mathrm{cr}}(2 \omega)+\mathbf{E}^{\mathrm{M}}(2 \omega)\right\}^{2}[13]$. Смена знака магнитного контраста ВГ вблизи резонанса ППП указывает на изменение фазы волны второй гармоники в резонансной области. Для сравнения была измерена аналогичная зависимость для подложки BIG, которая показала постоянство знака $\rho_{2 \omega}$ во всем исследованном угловом диапазоне.

Методом однолучевой интерферометрии ВГ при использовании тонкой пленки оксида индия-олова в качестве эталонного источника ВГ были проведены прямые измерения фазы магнитной и немагнитной составляющих волны ВГ. Измерения показали, что фазовый сдвиг обеих составляющих волны ВГ вблизи резонанса равен $\sim 2 \pi$, что соответствует поведению нелинейного резонанса Фано при резонансе на поле накачки. В то же время модуляция разности фаз магнитоиндуцированной и кристаллографических составляющих превышает $\pi / 2$, что соответствует наблюдаемому изменению знака магнитного контраста.

\section{4. Обсуждение результатов}

Значительное различие интенсивностных магнитооптических эффектов в линейном и нелинейном отклике обусловлено разными механизмами данных эффектов. В случае линейного отклика (прохождения) эффект наблюдается за счет смещения дисперсионных кривых для ППП при наличии намагниченности. С другой стороны, в нелинейном отклике (генерация второй гармоники) эффекты обусловлены сильной модуляцией фазы поля накачки при возбуждении ППП.

На основании полученных экспериментальных зависимостей предложена феноменологическая модель генерации ВГ в МПК, основанная на интерференции магнитной (от границы раздела $\mathrm{Au} / \mathrm{BIG}$ ) и кристаллографической (от границы раздела воздух/Au) компонент поля ВГ, испытывающих резонансный абсолютный $(2 \pi)$ и относительный (больше $\pi / 2$ ) сдвиг в окрестности резонансного возбуждения ППП на границе раздела $\mathrm{Au} / \mathrm{BIG}$. Взаимодействие этих двух полей ВГ определяет форму спектральной зависимости интенсивности ВГ в области ППП на границе $\mathrm{Au} / \mathrm{BIG}$ в виде нелинейного резонанса Фано, а также обнаруженного магнитного нелинейно-оптического эффекта.

\section{5. Заключение}

Таким образом, в работе представлены результаты исследования спектроскопии генерации второй оптической гармоники в магнитных плазмонных структурах на основе периодических массивов нанополос золота и феррита граната. Экспериментально показано, что в области резонансного возбуждения поверхностного плазмона на границе раздела металла и магнитного диэлектрика наблюдается резкая знакопеременная модуляция магнитного контраста ВГ, достигающая 40\% и сопровождающаяся изменением фазы волны ВГ на $2 \pi$. Полученные результаты описаны в терминах нелинейного резонанса Фано.

\section{Список литературы}

[1] V.V. Temnov, G. Armelles, U. Woggon, D. Guzatov, A. Cebollada, A. Garcia-Martin, J.M. Garcia-Martin, T. Thomay, A. Leitenstorfer, R. Bratschitsch. Nature Photon. 4, 107111 (2010).

[2] V.I. Belotelov, I.A. Akimov, M. Pohl, V.A. Kotov, S. Kasture, A.S. Vengurlekar, A.V. Gopal, D.R. Yakovlev, A.K. Zvezdin, M. Bayer. Nature Nanotechnol. 6, 370 (2011).

[3] A.A. Grunin, A.G. Zhdanov, A.A. Ezhov, E.A. Ganshina, A.A. Fedyanin. Appl. Phys. Lett. 97, 261908 (2010).

[4] G. Armelles, A. Cebollada, A. Garcia-Martın, J.M. GarciaMartın, M.U. Gonzalez, J.B. Gonzalez-Dıaz, E. Ferreiro-Vila, J.F. Torrad. J. Opt. A: Pure Appl. Opt. 11, 114023 (2009).

[5] A.L. Chekhov, V.L. Krutyanskiy, A.N. Shaimanov, A.I. Stognij, T.V. Murzina. Opt. Express 22, 17762 (2014).

[6] M. Pohl, L.E. Kreilkamp, V.I. Belotelov, I.A. Akimov, A.N. Kalish, N.E. Khokhlov, V.J. Yallapragada, A.V. Gopal, M. Nur-E-Alam, M. Vasiliev, D.R. Yakovlev, K. Alameh, A.K. Zvezdin, M. Bayer. New J. Phys. 15, 075024 (2013).

[7] A.L. Chekhov, V.L. Krutyanskiy, V.A. Ketsko, A.I. Stognij, T.V. Murzina. Opt. Mater. Express 5, 1647 (2015).

[8] J.Y. Chin, T. Steinle, T. Wehlus, D. Dregely, T. Weiss, V.I. Belotelov, B. Stritzker, H. Giessen. Nature Commun. 4, 1599 (2013).

[9] Y.R. Shen. The principles of nonlinear optics. WileyInterscience, N.Y. (1984). 563 p.

[10] V. Bespalova, O.L. Golikovaa, S.S. Savina, A.I. Stognij, N.N. Novitskii. Inorg. Mater. 48, 1190 (2012).

[11] B. Luk'yanchuk, N.I. Zheludev, S.A. Maier, N.J. Halas, P. Nordlander, H. Giessen, C.T. Chong. Nature Mater. 9, 707 (2010).

[12] A.K. Zvezdin, V.A. Kotov. Modern magnetooptics and magnetooptical materials. IOP Publ., Bristol (1997). 386 p.

[13] R.-P. Pan, H.D. Wei, Y.R. Shen. Phys. Rev. B 39, 1229 (1989). 